\title{
Article
}

\section{Galaxy and Mass Assembly (GAMA): probing the merger histories of massive galaxies via stellar populations}

Ferraras, I, Hopkins, AM, Gunawardhana, MLP, Sansom, Anne E, Owers, MS, Driver, S, Davies, L, Robotham, A, Taylor, EN, Konstantopoulos, I, Brough, S, Norberg, P, Croom, S, Loveday, J, Wang, L and Bremer, M

Available at http://clok.uclan.ac.uk/17626/

Ferraras, I, Hopkins, AM, Gunawardhana, MLP, Sansom, Anne E ORCID: 00000002-2782-7388, Owers, MS, Driver, S, Davies, L, Robotham, A, Taylor, EN et al (2017) Galaxy and Mass Assembly (GAMA): probing the merger histories of massive galaxies via stellar populations. Monthly Notices of the Royal Astronomical Society, 468 (1). pp. 607-619. ISSN 0035-8711

It is advisable to refer to the publisher's version if you intend to cite from the work. http://dx.doi.org/10.1093/mnras/stx503

For more information about UCLan's research in this area go to http://www.uclan.ac.uk/researchgroups/ and search for < name of research Group>.

For information about Research generally at UCLan please go to http://www.uclan.ac.uk/research/

All outputs in CLoK are protected by Intellectual Property Rights law, including Copyright law. Copyright, IPR and Moral Rights for the works on this site are retained by the individual authors and/or other copyright owners. Terms and conditions for use of this material are defined in the policies page. 


\title{
Galaxy and Mass Assembly (GAMA): probing the merger histories of massive galaxies via stellar populations
}

I. Ferreras, ${ }^{1 \star}$ A. M. Hopkins,${ }^{2}$ M. L. P. Gunawardhana, ${ }^{3,4}$ A. E. Sansom, ${ }^{5}$ M. S. Owers, ${ }^{2,6}$ S. Driver, ${ }^{7,8}$ L. Davies, ${ }^{7}$ A. Robotham, ${ }^{7}$ E. N. Taylor, ${ }^{9}$ I. Konstantopoulos, ${ }^{2}$ S. Brough, ${ }^{2}$ P. Norberg, ${ }^{3}$ S. Croom, ${ }^{10,11}$ J. Loveday, ${ }^{12}$ L. Wang ${ }^{13,14}$ and M. Bremer ${ }^{15}$

${ }^{1}$ Mullard Space Science Laboratory, University College London, Holmbury St Mary, Dorking, Surrey RH5 6NT, UK

${ }^{2}$ Australian Astronomical Observatory, PO Box 915, North Ryde, NSW 1670, Australia

${ }^{3}$ ICC and CEA, Department of Physics, Durham University, South Road, Durham DH1 3LE, UK

${ }^{4}$ Pontificia Universidad Católica de Chile, Vicuña Mackenna 4860, 7820436 Macul, Santiago, Chile

${ }^{5}$ Jeremiah Horrocks Institute, University of Central Lancashire, Preston PR1 2HE, UK

${ }^{6}$ Department of Physics and Astronomy, Macquarie University, Sydney, NSW 2109, Australia

${ }^{7}$ International Centre for Radio Astronomy Research, The University of Western Australia, 35 Stirling Hwy, Crawley, WA 6009, Australia

${ }^{8}$ SUPA, School of Physics \& Astronomy, University of St Andrews, North Haugh, St Andrews KY16 9SS, UK

${ }^{9}$ School of Physics, David Caro Building, The University of Melbourne, Parkville VIC 3010, Australia

${ }^{10}$ Sydney Institute for Astronomy (SIfA), School of Physics, The University of Sydney, NSW 2006, Australia

${ }^{11}$ ARC Centre of Excellence for All-sky Astrophysics (CAASTRO), 44-70 Rosehill Street, Redfern NSW 2016, Sydney, Australia

${ }^{12}$ Astronomy Centre, University of Sussex, Falmer, Brighton BN1 9QH, UK

${ }^{13}$ SRON Netherlands Insitute for Space Research, Landleven 12, NL-9747 AD Groningen, the Netherlands

${ }^{14}$ Kapteyn Astronomical Institute, University of Groningen, Postbus 800, NL-9700 AV Groningen, the Netherlands

${ }^{15} \mathrm{H}$ H Wills Physics Laboratory, Tyndall Avenue, Bristol BS8 1TL, UK

Accepted 2017 February 24. Received 2017 February 24; in original form 2016 November 18

\begin{abstract}
The merging history of galaxies can be traced with studies of dynamically close pairs. These consist of a massive primary galaxy and a less massive secondary (or satellite) galaxy. The study of the stellar populations of secondary (lower mass) galaxies in close pairs provides a way to understand galaxy growth by mergers. Here we focus on systems involving at least one massive galaxy - with stellar mass above $10^{11} \mathrm{M}_{\odot}$ in the highly complete Galaxy and Mass Assembly (GAMA) survey. Our working sample comprises 2692 satellite galaxy spectra $(0.1$ $\leq z \leq 0.3$ ). These spectra are combined into high S/N stacks, and binned according to both an 'internal' parameter, the stellar mass of the satellite galaxy (i.e. the secondary), and an 'external' parameter, selecting either the mass of the primary in the pair, or the mass of the corresponding dark matter halo. We find significant variations in the age of the populations with respect to environment. At fixed mass, satellites around the most massive galaxies are older and possibly more metal-rich, with age differences $\sim 1-2$ Gyr within the subset of lower mass satellites $\left(\sim 10^{10} \mathrm{M}_{\odot}\right)$. These variations are similar when stacking with respect to the halo mass of the group where the pair is embedded. The population trends in the lower mass satellites are consistent with the old stellar ages found in the outer regions of massive galaxies.
\end{abstract}

Key words: galaxies: evolution-galaxies: formation-galaxies: interactions-galaxies: stellar content.

\section{INTRODUCTION}

Galaxy growth is one of the fundamental processes linking structure formation and the observable Universe. The connection be- tween the evolution of (dark matter dominated) structures and the 'baryon physics' of galaxy formation is the 'holy grail' of extragalactic astrophysics. Galaxy mergers lead both to the mixing of the stellar component already in place in the progenitors, and to gas inflows that provide newly formed stars. In particular, massive galaxies are one of the best targets to put these processes to the test. At present, the most widely accepted theory for the formation of 
massive galaxies relies on a two-step scenario (e.g. Lackner et al. 2012; Oser et al. 2012). An early stage of collapse and efficient star formation in situ builds the core of massive galaxies, whereas the outer regions are populated by stars formed ex situ, incorporated in the galaxy during subsequent merging events. This theoretical scenario was motivated by the observations of compact, massive galaxies at high redshift (see e.g. Daddi et al. 2005; Trujillo et al. 2006, 2007; van Dokkum et al. 2010), in contrast with their more extended counterparts at lower redshift. Studies of the stellar populations in these compact systems reveal a growth mechanism driven by the accretion of the stellar components of companion galaxies through merging, ruling out significant levels of in situ star formation during this growth phase (Trujillo, Ferreras \& de La Rosa 2011).

Simulations provide grounds for this interpretation (Hirschmann et al. 2015), although there are still important aspects, such as the radial gradients in the age and chemical composition of the populations within galaxies, which require more work. Whilst the outer regions of massive galaxies are expected to originate mostly from minor mergers (Naab, Johansson \& Ostriker 2009), the old stellar ages found in the outskirts of massive early-type galaxies (La Barbera et al. 2012; Greene et al. 2015) reveal an important environment-related effect on the progenitor (minor-merging) systems. The stellar populations of satellites located dynamically close to massive galaxies are expected to be incorporated into the merged system. Therefore, a simple but effective way to study merging systems is based on samples of galaxies involving very close pairs in projected distance and relative velocity, i.e. those that would be expected to merge within a relatively short time (see e.g. Le Fèvre et al. 2000; Patton et al. 2000; Rogers et al. 2009; LópezSanjuan et al. 2012; Mármol-Queraltó et al. 2012, 2013; Newman et al. 2012; Conselice 2014). In Ferreras et al. (2014), a sample of close pairs at redshift $z \sim 0.5-1$ was explored via medium-band photometry (SHARDS; Pérez-González et al. 2013), serving as a low-resolution $(R \sim 50)$ spectrograph. The age of the populations in galaxies dynamically close to massive galaxies was found to obey the same mass-age relationship as galaxies in the field. That study was complete down to a 1:30 stellar mass ratio, suggesting that such a scenario would not be compatible with the flat age gradients found in massive ETGs, unless the net mass fraction provided by minor mergers was small. Furthermore, a number count analysis supported the idea that mass ratios closer to $\sim 1: 3$ dominate this growth channel in massive galaxies. An extension of this study to a very large sample of low- $z$ galaxies from Sloan Digital Sky Survey (SDSS) (Ruiz, Trujillo \& Mármol-Queraltó 2014) confirmed that mass ratios in the region 1:5 are more important than minor merging systems (typically defined by mass ratios below $1: 10$ ).

This project focuses on a more accurate determination of the properties of stellar populations, taking advantage of the high density of optical spectra available in Galaxy and Mass Assembly (GAMA, with respect to SDSS) to build a catalogue of galaxy spectra comprising dynamically close pairs with at least one massive galaxy (the DR2 GAMA catalogue already includes over 15000 galaxies with stellar mass above $10^{11} \mathrm{M}_{\odot}$ ). These pairs are the progenitors of merged systems, and via comparisons with numerical simulations (see e.g. Kitzbichler \& White 2008; Jiang, Jing \& Han 2014), it is possible to derive the actual merger rates as a function of the mass ratio. Moreover, the selection of pairs involving at least a massive galaxy probes the regime where most of the growth proceeds via the accretion of lower mass galaxies (Robotham et al. 2014). This paper aims at measuring the properties of the stellar populations of the merger progenitors with respect to stellar mass, mass ratio and environment. The targeted close pairs are expected to merge at later times, mixing up their stellar populations. Depending on the dynamical characteristics of the system (mainly the merger mass ratio), it is possible to infer the properties of the radial gradients of stellar population properties in massive galaxies at later times (e.g. La Barbera et al. 2011). This work complements the recent GAMA-based study of Davies et al. $(2015,2016)$, devoted to the trends of star formation diagnostics in close pairs.

The methodology involves a careful stacking of observed spectra - binned according to common characteristics such as stellar mass or merger mass ratio - in order to reach the high Signal to Noise Ratio (SNR) needed to explore a set of line strength indices dependent on the age, metallicity and $[\alpha / \mathrm{Fe}]$ of the populations. We note that due to flux calibration issues in the GAMA/Anglo-Australian Telescope (AAT) spectra, it is better to probe the stellar populations via indices, avoiding spectral fitting (except for the narrow spectral window straddling the $4000 \AA$ A break). Note that the level of merging, derived from the analysis of close pairs, gives a fairly constant rate out to $z \sim 1.5$, quantified as a stellar mass growth inverse time-scale of $\tau^{-1}$ $\equiv(\Delta M / M) / \Delta t=0.08 \pm 0.02 \mathrm{Gyr}^{-1}$ (Ferreras et al. 2014). Over the redshift probed in this sample $(z \leq 0.3)$, one would thus expect a fractional stellar mass growth from mergers between 10 per cent (at $z=0.1$ ) and 30 per cent (at $z=0.3$ ). The analysis of the stellar populations of the satellite galaxies will impose valuable constraints on models of galaxy formation and evolution (e.g. Hirschmann et al. 2015).

The structure of the paper is as follows: Section 2 presents the data set extracted from the GAMA survey. Section 3 describes how the spectra are prepared and stacked, followed by Section 4 , devoted to the methodology regarding the analysis of the line strengths. A discussion section (Section 5) puts the results in context with our understanding of galaxy growth mechanisms, concluding with a summary in Section 6. A standard $\Lambda$ cold dark matter cosmology is adopted, with $\Omega_{\mathrm{m}}=0.27$ and $H_{0}=70 \mathrm{~km} \mathrm{~s}^{-1} \mathrm{Mpc}^{-1}$. As a reference, the look-back time to $z=0.2$ (the median of our working sample) is $2.4 \mathrm{Gyr}$ and 1 arcsec maps into $3.3 \mathrm{kpc}$ at that redshift.

\section{DATA}

We retrieve our sample from the GAMA-II data base, a panchromatic galaxy survey providing a set of spectroscopic redshifts down to $r_{\mathrm{AB}}=19.8 \mathrm{mag}$ (Liske et al. 2015). We focus on the equatorial fields, which cover $\sim 180 \mathrm{deg}^{2}$ in three regions, with a high ( $~ 98.5$ per cent) spatially uniform redshift completeness that makes it optimal for studies of environment (see e.g. Robotham et al. 2011; Brough et al. 2013). We note that in GAMA, the same fields were repeatedly visited, so that, by construction, the spectroscopic completeness is very high, not only in general, but also over small scales, avoiding the standard issues found in SDSS spectroscopic data sets regarding fibre collision. The tiling and observing strategies of the survey are discussed in detail in Robotham et al. (2010) and Driver et al. (2011).

Our selection starts with the general set of massive galaxies, defined as those with a stellar mass above $10^{11} \mathrm{M}_{\odot}$. The sample is extracted from the latest version (v18) of the catalogue of stellar masses in the GAMA survey (Taylor et al. 2011), and restricted to the $0.1 \leq z \leq 0.3$ redshift range, in order to minimize aperture effects. The set comprises 12616 massive galaxies. ${ }^{1}$ Within this

\footnotetext{
${ }^{1}$ From which 8186 sources have AAT spectra, and 4313 have SDSS spectra. The remaining 117 spectra were compiled from other surveys (2dFGRS, WiggleZ, etc.).
} 
sample of massive galaxies, we look for dynamically close pairs, which serve as potential merger progenitors. A close pair is defined here by a system separated by a projected distance less than $100 \mathrm{kpc}$, and with a velocity difference below $700 \mathrm{~km} \mathrm{~s}^{-1}$. Hereafter, we refer to the most massive galaxy in the pair as a primary, and the companion is termed either a secondary, or a satellite. This criterion yields a total of 3770 satellites, in 2787 systems (note that some of the primary galaxies may have more than one satellite). From these, only 227 satellite galaxies have SDSS spectra - as expected for such close pair systems, whereas AAT data are available for 3506 satellites. We want to minimize potential biases from systematics related to the use of spectra from different instruments. Given the higher completeness of the AAT spectra, we decide to use only these data in the analysis. Furthermore, we remove low-quality data from the sample, discarding all spectra with a low value of $n_{\mathrm{Q}}(<3$, as defined in Driver et al. 2011), or a low S/N ( $<3$, as defined by the RUNZ code). Moreover, a small fraction ( 2 per cent) of the GAMA/AAT spectra have severe fringing (Hopkins et al. 2013). Therefore, we also remove those visually inspected to feature such fringing, resulting in a final working sample of 2692 satellite spectra. The same criteria applied to the selection of massive galaxies yields a sample of 7702 systems.

\subsection{Sample selection effects}

The GAMA/AAT spectra were acquired through optical fibres that map on to a 2 arcsec-diametre aperture (Hopkins et al. 2013). Within the redshift range of the sample $(0.1 \leq z \leq 0.3)$, the fibre aperture extends over a projected physical distance between 3.7 and $8.9 \mathrm{kpc}$. Fig. 1 shows the distribution of our satellite galaxies with respect to the fibre aperture (measured in units of the effective radius, top panel); the Sérsic index (middle panel) and the redshift (bottom panel). The figure makes use of the surface brightness fits to the SDSS imaging of the GAMA fields from Kelvin et al. (2012). The results are colour-coded to easily detect any potential bias related to aperture effects or redshift. The diagrams show the typical trends expected with the stellar mass of the satellite (i.e. a horizontal colour gradient in this figure). The lack of galaxies in the bottom righthand corner of each panel is caused by the fact that by construction, $M_{\mathrm{PRI}}>M_{\mathrm{SAT}}$. Note that lower mass galaxies dominate the sample at low redshift and at lower Sérsic indices. Regarding the size of the galaxy with respect to the fibre size, there is a competing effect between the small intrinsic sizes of lower mass galaxies, and the small relative sizes of the more massive galaxies, preferentially located at higher redshift. This explains why in the top panel of Fig. 1 the largest values of relative size occur at stellar masses around $\log \left(M_{\mathrm{s}} / 10^{11} \mathrm{M}_{\odot}\right) \sim-0.5$. In any case, note that most of the spectra enclose the light within, at least, an effective radius. Therefore, the variations found in the spectra are expected to map the general population in these galaxies, and not potential radial gradients. Furthermore, note that in this paper, we focus on a differential analysis between satellite galaxies, at fixed stellar mass, with respect to either the mass of the primary or the mass of the halo where the system is located. We conclude that no significant systematic trend is expected from the sample selection or the use of optical fibres.

Fig. 2 shows the distribution of satellite galaxies with respect to their relative velocity $\left(\Delta v_{\mathrm{PEC}}\right)$ and projected separation $\left(\Delta R_{\perp}\right)$. The whole sample is shown as filled dots. In particular, red and blue dots correspond to galaxies with the highest $\left(M_{c}>2 \times 10^{11} \mathrm{M}_{\odot}\right)$ and lowest $\left(10^{11} \mathrm{M}_{\odot}<M_{c}<1.5 \times 10^{11} \mathrm{M}_{\odot}\right)$ values of the mass of the primary, respectively. No systematic differences are found in this diagram, although we note a steep decrease in the number of
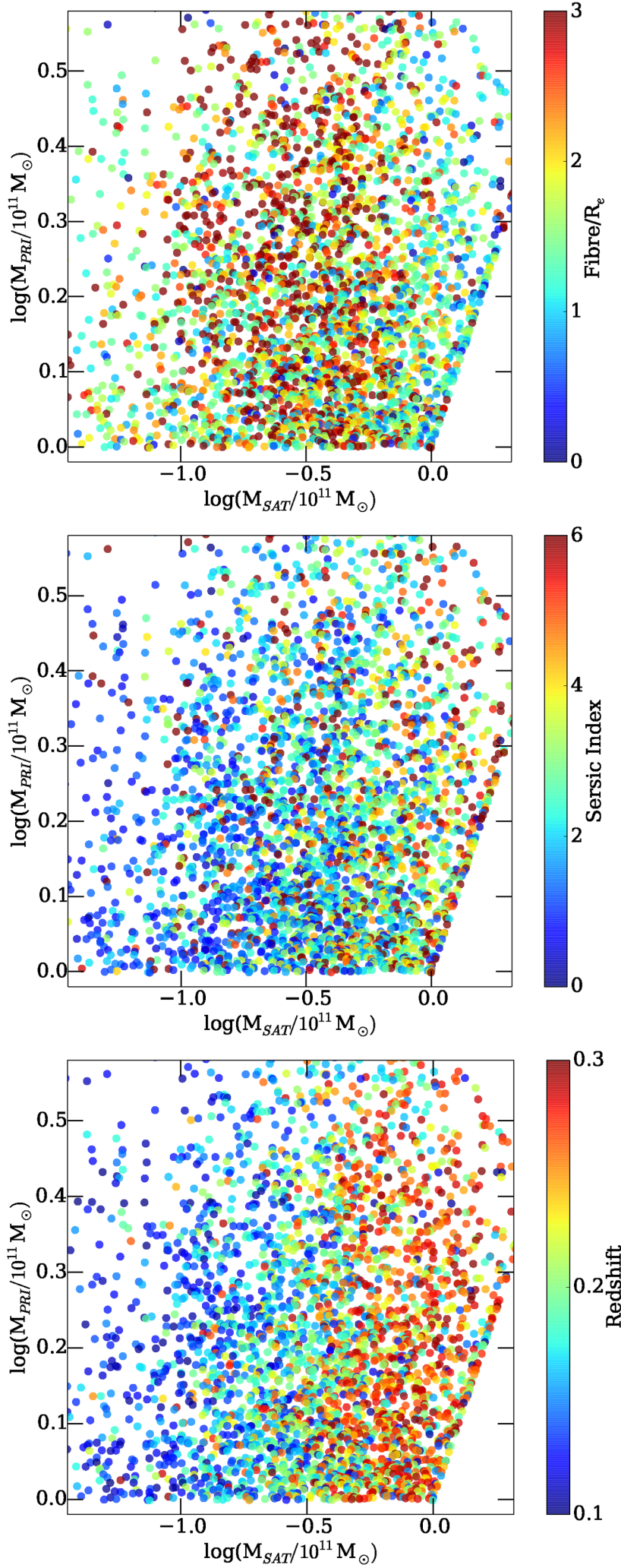

Figure 1. Colour-coded plots representing the sample on the primary versus secondary stellar mass. Colour represents fibre aperture (in units of the effective radius, top panel); Sérsic index (middle panel); and redshift (bottom panel). Note that a bias with respect to these quantities would show up as a significant colour trend (see the text for details). 


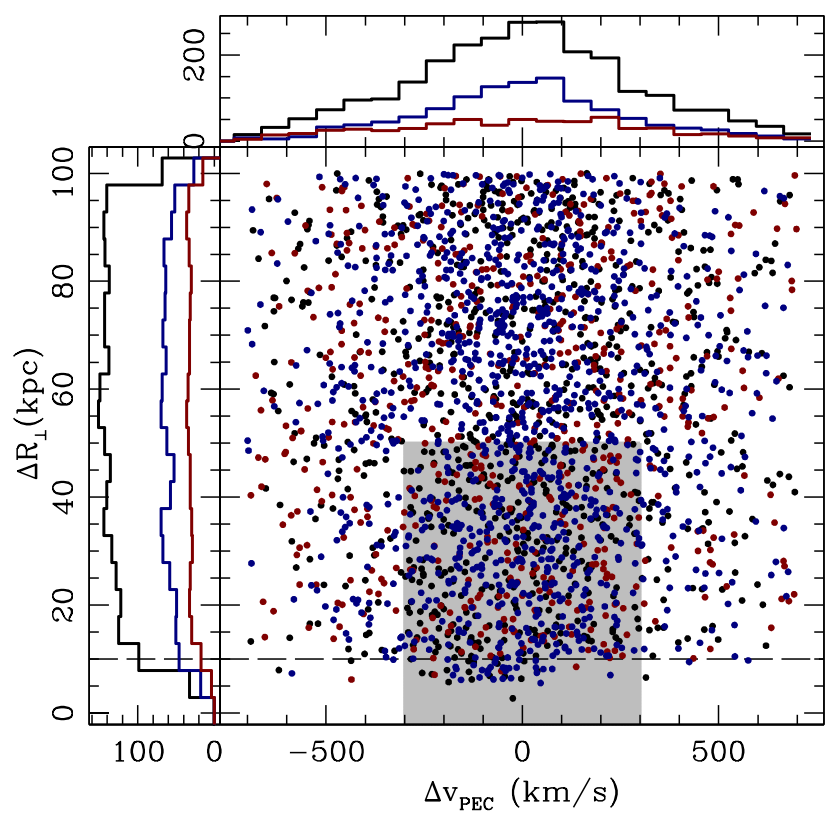

Figure 2. Distribution of the sample with respect to relative velocity ( $\left.\Delta v_{\text {PEC }}\right)$ and projected physical separation $\left(\Delta R_{\perp}\right)$. The red (blue) dots and histograms correspond to the subsample of satellites around the highest (lowest) mass primary galaxies. The horizontal dashed line marks the $\Delta R$ $=10 \mathrm{kpc}$ lower limit imposed on the satellite sample. The shaded region defines an additional subset of tighter galaxy pairs (see the text for details).

targets with $\Delta R_{\perp}<10 \mathrm{kpc}$ (horizontal dashed line). Therefore, we remove those satellites from the analysis of the general sample. In addition to the subsamples segregated with respect to primary mass, we also consider an additional subset made up of very close pairs, as shown by the grey shaded area in the figure. Pairs in this region $\left(\left|\Delta v_{\mathrm{PEC}}\right|<300 \mathrm{~km} \mathrm{~s}^{-1} ; 0<\delta R_{\perp}<50 \mathrm{kpc}\right)$ provide a more direct representation of merging progenitors.

\section{PREPARING THE SAMPLE FOR ANALYSIS}

\subsection{Individual spectra}

Individual spectra are retrieved from the GAMA-II data base and are corrected for foreground extinction using the colour excess maps of Schlafly \& Finkbeiner (2011), following a standard dust extinction law for the Milky Way (Cardelli, Clayton \& Mathis 1989). Given the typically low values of extinction on the footprint of the GAMA survey, this step is only - mildly - relevant for the derivation of the $4000 \AA$ break, whereas the rest of the analysis is based on a continuum-subtracted spectrum. The GAMA/AAT spectra present some flux calibration issues, most notably a variable level of fringing, and scattered light in the blue arm (see Hopkins et al. 2013, for details). We performed a number of tests involving spectral fitting, which gave us a complex range of residuals in the spectra that were not trivial to eliminate based on simple prescriptions. Therefore, in order to avoid any systematics related to flux calibration residuals, we restrict the analysis of stellar populations to absorption line features, performing a careful subtraction of the continuum, effectively removing any flux calibration problem.

The pseudo-continuum is defined following a robust method laid out in Rogers et al. (2010). In a nutshell, the pseudo-continuum is defined as a high-order percentile of the flux density values within a kernel window. At the resolution and sampling of SDSS spectra (similar, but not identical to GAMA data), Rogers et al. (2010) concluded that the continuum was best fit with a 90 per cent level within a $100 \AA$ window. This choice is not critical. We note that similar values were found independently for SDSS spectra of stars (Hawkins et al. 2014). In this case, we follow a three-step approach to derive the pseudo-continuum, with an initial pass with a $100 \AA$ window at the 90 per cent level, followed by a second pass on the derived continuum, with a $200 \AA$ window and a 90 per cent level. This pseudo-continuum is subtracted from the observed spectrum. A final, third step fits the derived continuum with a third-order polynomial that removes any residual changes over large scales in wavelength space. Such a technique has already been successfully applied to GAMA/AAT spectra (Baldry et al. 2014). All the spectra are normalized to the same flux within the $[4400,4800] \AA$ wavelength range in the rest-frame.

In addition, we include in the analysis the strength of the $4000 \AA$ break, $D_{n}(4000)$, as defined by Balogh et al. (1999). This feature measured prior to continuum removal - extends over a $250 \AA$ A region, and so, we expect the results not to be significantly affected by flux calibration issues. In contrast to the other line strengths, this index is measured on individual spectra, and the values corresponding to each set of stacked spectra are combined to provide the average and uncertainty of the index of a given stack.

\subsection{Stacking procedure}

Our analysis is based on spectral line strengths to constrain the age and chemical composition of the populations. Such an approach requires relatively high $\mathrm{S} / \mathrm{N}$, leading us to stack spectra, discarding the very noisy ones. Our final working sample only includes spectra with $\mathrm{S} / \mathrm{N}$ (as defined by the RUNZ code) above 3 . We illustrate the selection of individual spectra for stacking in Fig. 3, where the orange lines delimit the binning scheme. We use the stellar mass of the secondary as the 'local' parameter (horizontal axis). The 'environment' parameter is defined either as the mass of the primary (left-hand panel), or the mass of the group within which the pair live (middle and right-hand panels). The groups are defined in the $\mathrm{G}^{3} \mathrm{C}$ catalogue of Robotham et al. (2011, we use v09), and are translated into halo masses following either the scaling relation with total luminosity from Viola et al. (2015), or the dynamically based halo masses of Robotham et al. (2011), from derived estimates of the size and velocity dispersion of the group. For the latter, we use their mass proxy corrected by the $A$ factor. The quantity $\mu \equiv M_{\mathrm{SAT}} / M_{\mathrm{PRI}}$ can be interpreted as the mass ratio of the eventual merging system.

The grey dots in Fig. 3 correspond to secondary galaxies with available GAMA/AAT spectra. As a reference, two values of the mass ratio $(1: 1$ and $1: 10$, corresponding to $\mu=1$ and 0.1 , respectively) are shown as blue dashed lines on the left-hand panel. Table 1 shows the number of spectra used for the analysis presented in this paper. Note that not all of the galaxies from the close pair sample are listed in the groups catalogue. In addition, we consider two additional samples, a subset of 'very close' pairs - as defined by the shaded region in Fig. 2 - consisting of systems with a small relative velocity and projected separation, and a large sample made up of all GAMA/AAT spectra with the same constraints with respect to $\mathrm{S} / \mathrm{N}$, redshift, etc., but regardless of whether they are close to a massive galaxy. This sample will help to compare the properties of the satellite galaxies with respect to the general population. This larger sample is hereafter termed the field sample. Table 2 shows the number and $\mathrm{S} / \mathrm{N}$ of these two additional sets.

After following the steps described in Section 3.1 for the individual spectra, the continuum-subtracted data are stacked, with a 

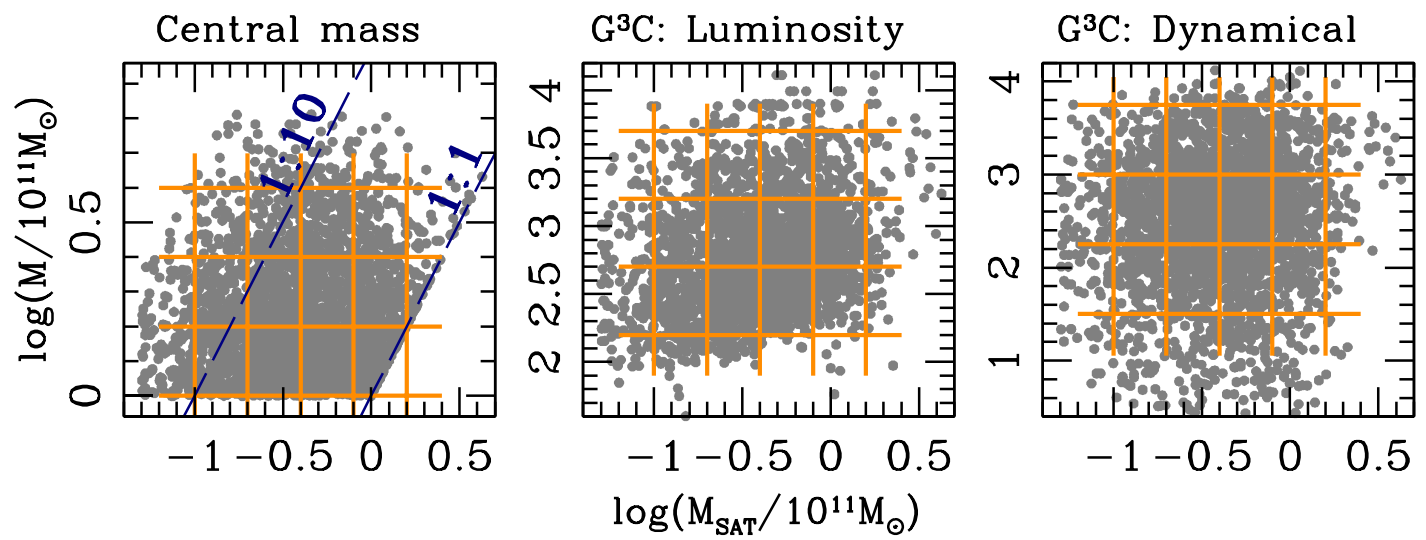

Figure 3. Grids defining the stacking procedure: This diagram shows the stellar mass of the primary and secondary galaxies for each of the close pairs defined in the working sample. The grey dots correspond to the GAMA/AAT spectra. The orange grids represent the regions that define the $4 \times 3$ stacked spectra. We define a local parameter, defined by the stellar mass of the secondary galaxy, and an environment parameter defined either by the mass of the most massive companion (called the primary galaxy, left-hand panel), by the luminosity-derived group mass (middle panel), or the dynamical group mass (right-hand panel). Group masses are retrieved from the $\mathrm{G}^{3} \mathrm{C}$ catalogue of Robotham et al. (2011), and the luminosity-derived estimates follow the scaling relation of Viola et al. (2015). For reference, the blue dashed lines on the left panel show the loci for a 1:1 and a 1:10 merger progenitor.

Table 1. Number and $\mathrm{S} / \mathrm{N}$ (in brackets) of spectra used in the stacks (see Fig. 3). The $\mathrm{S} / \mathrm{N}$ is given per pixel, averaged in the region around the $\mathrm{Mgb}$ feature $(\lambda \sim 5175 \AA)$. The group halo mass is derived either from the total luminosity given by the $\mathrm{G}^{3} \mathrm{C}$ catalogue of Robotham et al. (2011), following the scaling of Viola et al. (2015), or by the dynamical estimate presented in Robotham et al. (2011), as labelled.

\begin{tabular}{|c|c|c|c|c|}
\hline \multirow[b]{2}{*}{$\log M_{\mathrm{SAT}} / \mathrm{M}_{\odot}$} & \multicolumn{4}{|c|}{$\log M_{\mathrm{PRI}} / \mathrm{M}_{\odot}$ stacks } \\
\hline & $11.0-11.2$ & $11.2-11.4$ & $11.4-11.6$ & ALL \\
\hline $10.0-10.3$ & $217(72)$ & 164(57) & $58(45)$ & $439(102)$ \\
\hline $10.3-10.6$ & $391(118)$ & 254(92) & $114(75)$ & $759(167)$ \\
\hline $10.6-10.9$ & $440(144)$ & $262(116)$ & $132(101)$ & $834(211)$ \\
\hline \multirow[t]{2}{*}{$10.9-11.2$} & $215(122)$ & $171(134)$ & $74(100)$ & $460(207)$ \\
\hline & \multicolumn{4}{|c|}{$\log M_{\mathrm{GRP}, \mathrm{L}} / \mathrm{M}_{\odot}$ stacks: luminosity-based } \\
\hline $\log M_{\mathrm{SAT}} / \mathrm{M}_{\odot}$ & $13.2-13.7$ & $13.7-14.2$ & $14.2-14.7$ & ALL \\
\hline $10.0-10.3$ & $247(61)$ & $119(34)$ & $35(19)$ & $401(72)$ \\
\hline $10.3-10.6$ & $371(96)$ & $258(73)$ & $74(38)$ & $703(127)$ \\
\hline $10.6-10.9$ & $349(124)$ & $295(104)$ & $114(62)$ & $758(173)$ \\
\hline \multirow[t]{2}{*}{$10.9-11.2$} & $124(102)$ & $218(135)$ & $69(70)$ & $411(183)$ \\
\hline & \multicolumn{4}{|c|}{$\log M_{\mathrm{GRP}, \mathrm{D}} / \mathrm{M}_{\odot}$ stacks: dynamical } \\
\hline $\log M_{\mathrm{SAT}} / \mathrm{M}_{\odot}$ & $12.50-13.25$ & $13.25-14.00$ & $14.00-14.75$ & ALL \\
\hline $10.0-10.3$ & $142(46)$ & 176(49) & $83(25)$ & $401(72)$ \\
\hline $10.3-10.6$ & $229(73)$ & $308(90)$ & $169(54)$ & 706 (127) \\
\hline $10.6-10.9$ & 242(98) & 317 (115) & 203(87) & $762(174)$ \\
\hline $10.9-11.2$ & $112(102)$ & $194(125)$ & 102(85) & 408 (183) \\
\hline
\end{tabular}

weighting scheme following a sigmoid function with a threshold at $\mathrm{SNR}=8$, namely

$w(\mathrm{SNR})=1-\frac{1}{\mathrm{e}^{\mathrm{SNR}-8}+1}$,

where SNR is the average SNR per $\AA$ within the rest-frame interval 4000-4300 $\AA$. This scheme weighs equally all data points within the same individual spectrum. We note that the standard scheme, using the inverse variance as a weight, gives slightly noisier stacks. The resulting spectra are corrected for nebular emission. We perform spectral fitting using the code STARLIGHT (Cid Fernandes et al. 2005), using as basis functions a set of 92 model
Table 2. Number and $\mathrm{S} / \mathrm{N}$ (in brackets) of additional spectra used here. The $\mathrm{S} / \mathrm{N}$ is computed on the stacked data.

\begin{tabular}{lcc}
\hline $\log M_{\mathrm{SAT}} / \mathrm{M}_{\odot}$ & Number $(\mathrm{S} / \mathrm{N})$ \\
\hline & Close sample $^{1}$ & \\
$10.0-10.3$ & & $108(48)$ \\
$10.3-10.6$ & $212(89)$ \\
$10.6-10.9$ & $238(121)$ \\
$10.9-11.2$ & $181(133)$
\end{tabular}

Field sample ${ }^{2}$

\begin{tabular}{ll}
$10.0-10.3$ & $14631(1409)$ \\
$10.3-10.6$ & $21705(2010)$ \\
$10.6-10.9$ & $22044(2403)$ \\
$10.9-11.2$ & $10659(2162)$ \\
\hline
\end{tabular}

Notes. ${ }^{1}$ Defined by $\Delta v_{\mathrm{PEC}}<300 \mathrm{~km} \mathrm{~s}^{-1}, \Delta R_{\perp}$ $<50 \mathrm{kpc}$.

${ }^{2}$ Comprises all GAMA/AAT spectra with the same constraints as in the close pair sample, except for the proximity to a massive galaxy.

spectra from Vazdekis et al. (2012), after applying the same continuum normalization as on the observed spectra. The basis functions cover a wide range of ages - from 0.3 to $13.7 \mathrm{Gyr}$ in 23 steps in logarithmic space, and metallicity - from $\log \left(Z / Z_{\odot}\right)=-0.5$ to +0.2 in 4 steps, logarithmic with respect to $Z$. The output gives the effective velocity dispersion of the stack, and a best-fitting spectrum. Fig. 4 shows the procedure in two stacks, where the difference between the observed spectra and the best-fitting model (bottom panels) reveals the presence of the emission lines. These lines $(\mathrm{H} \beta, \mathrm{H} \gamma, \mathrm{H} \delta,[\mathrm{O} \mathrm{III}])$ are fit in the residual, using Gaussian profiles, following a Levenberg-Marquardt algorithm. These fitted lines are removed from the original stack and the final, emission-corrected spectrum is smoothed to a fiducial velocity dispersion of $200 \mathrm{~km} \mathrm{~s}^{-1}$, using a Gaussian kernel. The line strengths are measured on the final spectra, using the uncertainties in the stacks to bootstrap the errors on the equivalent widths from an ensemble of 100 realizations. 


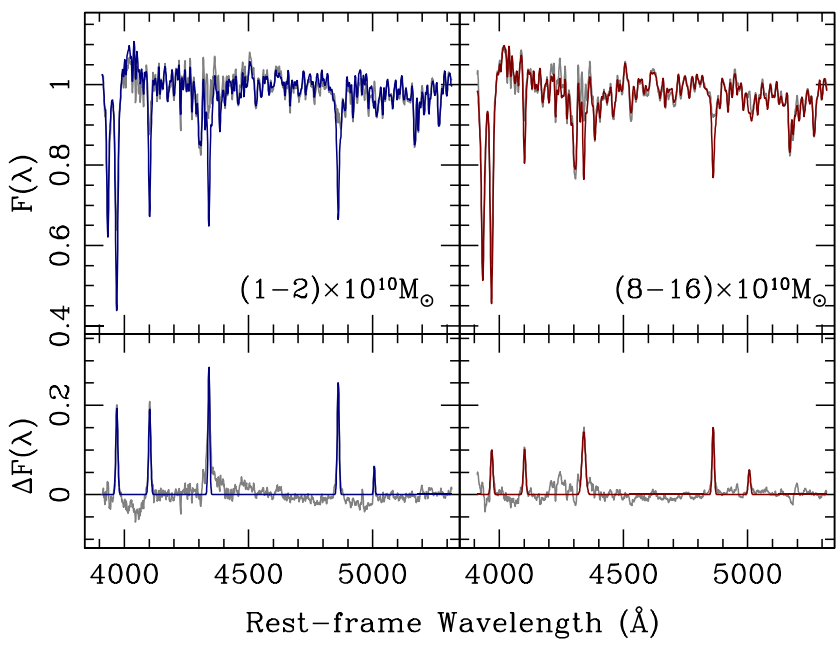

Figure 4. Illustration of the emission-line correction of the stacked spectra. The left-hand (right-hand) panels correspond to the stacked spectra of satellite galaxies with the lowest (highest) stellar mass. The top panels plot the original stack (in grey) and the best-fitting model (in blue/red colour). The bottom panels show the difference between the original and the best-fitting model (in grey) and between the original and the final, cleaned spectra (in blue/red colour).

\section{METHODOLOGY}

Fig. 5 shows the continuum-subtracted stacks from our sample when the 'environment' parameter is defined as the stellar mass of the primary. From bottom to top, the sample is shown in increasing

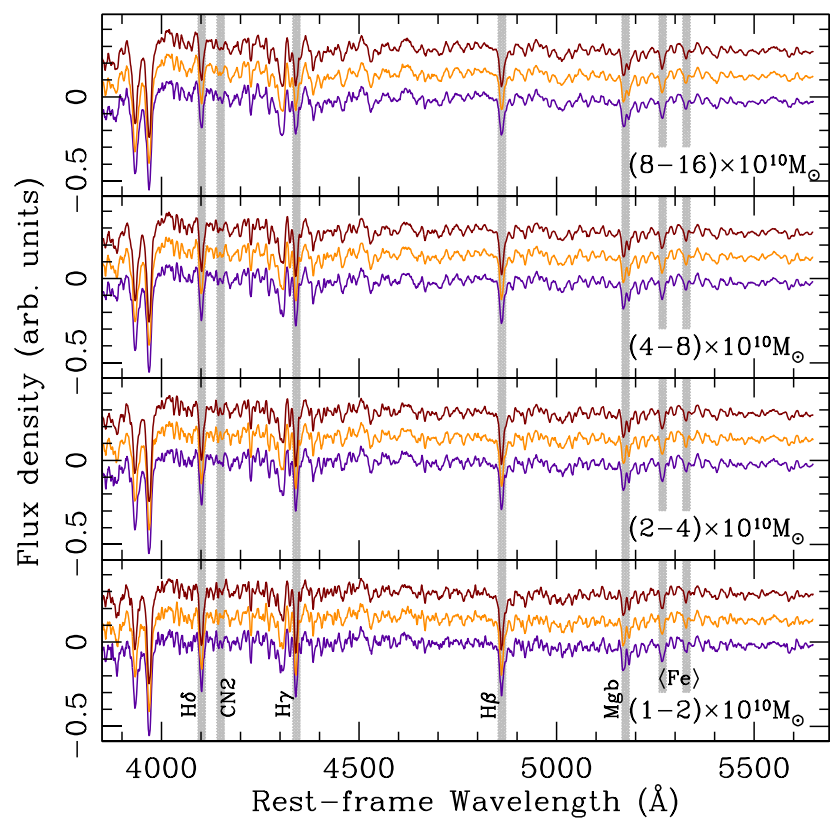

Figure 5. Continuum-subtracted stacked spectra of the satellite galaxies. From bottom to top, the different panels correspond to increasing values of the stellar mass of the satellite, as labelled. Within each panel, three stacks are shown, in red, orange and blue, corresponding to decreasing values of the stellar mass of the primary - as shown in the grids of Fig. 3. The spectra have been corrected for emission in the Balmer lines (see the text for details). For ease of visualization, the spectra have been shifted arbitrarily along the vertical direction (flux). The grey shaded areas mark typical spectral features used in the analysis, from blue to red: $\mathrm{H} \delta, \mathrm{CN} 2, \mathrm{H} \gamma, \mathrm{H} \beta, \mathrm{Mgb}, \mathrm{Fe} 5270$ and Fe5335. stellar mass of the satellite, as labelled, whereas in each panel, three spectra are shown, corresponding from bottom to top to an increased mass of the primary (see grids on the left-hand panel of Fig. 3 for reference). The spectra are shifted vertically by a constant amount for clarity. The vertical shaded regions encompass the line strengths used in the analysis. Note the significant variation in effective resolution with respect to satellite mass, caused by the velocity dispersion of the stars, especially evident in the Mgb feature at $\lambda \sim 5170 \AA$. This trend is removed from the analysis by smoothing all the spectra to a common fiducial value of $200 \mathrm{~km} \mathrm{~s}^{-1}$.

The continuum-subtracted stacked spectra provide information about the underlying stellar populations of the putative progenitors of massive galaxies. We can therefore explore differences in targeted line strengths to probe the characteristics of the populations that will be eventually incorporated in massive galaxies at later times. Although the details of the process vary substantially from system to system, we can generally assume that in minor merging systems, the populations of the satellite galaxy will be incorporated in the outer envelope of the merged system (see e.g. Naab et al. 2009), whereas a major merger will produce a more efficient spatial mixing of both galaxies.

The analysis is performed by comparing the observed, stacked data with population synthesis models. We choose the latest version of the MIUSCAT models from Vazdekis et al. (2012). These models provide the spectra of simple stellar populations at a $2.51 \AA$ resolution (full width at half-maximum; Falcón-Barroso et al. 2011), over the $\lambda \lambda 3465-9469 \AA$ spectral window for a range of ages, metallicities and stellar initial mass functions (IMF). We note that in our analysis (restricted to rest-frame wavelengths bluer than $5500 \mathrm{AA}$ ), the MIUSCAT models are fully based on the MILES stellar library (Sánchez-Blázquez et al. 2006). For simplicity, we adopt the standard Kroupa-universal IMF (Kroupa 2001). The synthetic spectra are processed following the identical methodology as the GAMA/AAT spectra, after being convolved with a Gaussian kernel to the fiducial velocity dispersion of $200 \mathrm{~km} \mathrm{~s}^{-1}$ chosen for all the stacked satellite spectra. The observed $\left(\left\{o_{i} \pm \sigma_{i}\right\}\right)$ and model $\left(\left\{m_{i}\right\}\right)$ line strengths are compared with a standard $\chi^{2}$ statistic:

$\chi^{2}(t, Z) \equiv \sum_{i}\left[\frac{o_{i}-m_{i}(t, Z)}{\sigma_{i}}\right]^{2}$,

where the indices used in the analysis are: $\left\{o_{i}\right\}=$ $\left\{\mathrm{H} \beta_{o}, \mathrm{H} \gamma_{\mathrm{F}}, \mathrm{H} \delta_{\mathrm{F}}, \mathrm{CN} 2, D_{n}(4000),[\mathrm{MgFe}]^{\prime}\right\}$, comprising the standard age-sensitive Balmer lines: $\mathrm{H} \beta_{\mathrm{o}}-$ following the definition of Cervantes \& Vazdekis (2009), $\mathrm{H} \gamma_{\mathrm{F}}$ and $\mathrm{H} \delta_{\mathrm{F}}$ (Worthey \& Ottaviani 1997). The CN2 index (Trager et al. 1998) is also included, as previous work in the literature reports variations with respect to environment (Carretero, Vazdekis \& Beckman 2007) and could be potentially used as a stellar clock in addition to $[\mathrm{Mg} / \mathrm{Fe}]^{\prime}$. We include in the analysis the $4000 \AA$ break strength (Balogh et al. 1999), and the standard metallicity-sensitive indices: $\mathrm{Mgb},\langle\mathrm{Fe}\rangle=\mathrm{Fe} 5270+\mathrm{Fe} 5335$ and $[\mathrm{MgFe}]^{\prime}$ (Thomas, Maraston \& Bender 2003). We note that although these line strengths follow the standard definitions of the index and the red/blue sidebands, they are measured on continuum-subtracted data, except for $D_{n}(4000)$.

The model data are derived from a grid of 1024 Simple Stellar Population (SSP) models, taking 16 steps in metallicity, from $\log \left(Z / Z_{\odot}\right)=-0.7$ to +0.2 and 64 log-steps in age, from 0.3 to 13.7 Gyr. Note that in order to obtain an accurate estimate of SSPequivalent ages and metallicities, this grid is much denser than the one used in Section 3.2 to obtain a best-fitting spectrum to correct for the effect of emission lines. We do not use $\mathrm{Mgb}$ or $\langle\mathrm{Fe}\rangle$ in 
the analysis, as the combined index, $[\mathrm{MgFe}]^{\prime}$, already provides the constraint on the metallicity, independently of $[\alpha / \mathrm{Fe}]$ enhancement (Thomas et al. 2003). Since we are using a reduced set of measurements, we only consider SSP-equivalent variations. Although we warn that constraints based on SSPs need not provide an accurate estimate of absolute ages and metallicites, differential variations in stellar populations are captured quite accurately by SSP-equivalent parameters (see e.g. Rogers et al. 2010). Furthermore, the added degeneracies inherent to the use of composite populations may wash out variations in the spectral features.

\section{DISCUSSION}

Fig. 6 shows the trend in the equivalent widths (EWs) of the ageand metallicity-sensitive features, as a function of the stellar mass of the satellite galaxies. We emphasize here that the aim is to look for differences in the properties of satellites close to massive galaxies - whose stellar populations will be eventually merged. Three sets are presented corresponding to the stacking criteria discussed above. The top panels (labelled A) show the EWs when stacking according to the stellar mass of the primary: The red (blue) lines correspond to satellites around the most (least) massive primary galaxy. In addition, we show in orange the EWs for the subsample of satellites within a closer range of the massive galaxy (i.e. the shaded region in Fig. 2). The middle and bottom panels (labelled $\mathrm{B}$ and $\mathrm{C}$ ) show the results for the alternative stacking procedure, based on the halo mass of the hosting groups. In this case, the red (blue) colours correspond to galaxies lying in the most (least) massive groups. The difference between the middle and bottom panels lie in the definition of halo mass: (B) uses the luminosity-derived masses from Viola et al. (2015), whereas (C) uses the dynamical estimates from Robotham et al. (2011) (see Section 3.2). In all three sets $(\mathrm{A}-\mathrm{C})$, the grey shaded regions extend over the range of the satellite sample in general, i.e. only stacked with respect to satellite mass, regardless of primary mass or group mass. The dotted lines in both sets are the results for the larger field sample, i.e. not restricted to the presence of a close pair, but with the same redshift distribution.

The EWs of the stacked satellite spectra reveal a significant difference in the stellar populations at fixed stellar mass, so that the satellites of the most massive primary galaxies (red lines) are slightly older and more metal-rich. In addition, note that the general sample of field galaxies (dotted lines) feature younger and metal-poorer populations at fixed stellar mass. Therefore, there is a significant environment-related trend where star formation proceeded more efficiently when satellites are located in the proximity of a more massive galaxy. One could argue that this is a group-related trend, so that satellites close to the most massive galaxies tend to live in more massive haloes. Moreover, galactic conformity (Weinmann et al. 2006) poses that the properties of galaxies within a group correlate with the properties of the central galaxy sitting at the centre of the dark matter halo. Therefore, we should consider whether the observed trend is either caused by the short-range effect of being in a close pair, or, rather, by group-related mechanisms. The second set of panels (B and C) - corresponding to group-based stacking gives similar trends when considering halo masses. We also note that since the total luminosity in a group has a stronger correlation with the luminosity of the central galaxy, it is difficult to disentangle (central) galaxy mass and (luminosity-derived) halo mass, whereas dynamical masses are derived from independent estimates of the halo properties, such as size or velocity dispersion. Of the various ways of measuring halo mass, the one based on the cor- relation with total luminosity is supposed to be less biased than the dynamical estimates (Han et al. 2015). Hartley et al. (2015) explored galactic conformity at higher redshift $(z \lesssim 2)$ finding no significant evolution with cosmic time. They concluded that a halo mass-independent mechanism could be responsible for this trend, such as a hot halo produced by the massive companion (cf. Kawinwanichakij et al. 2016). In this context, our trends cannot disentangle the difference between the effect of the primary or a potential mechanism caused by the interaction with the halo where the pair is embedded.

We need to test whether these trends are representative of the observed sample. Fig. 7 shows the distribution of a few line strengths measured on individual spectra, corresponding to satellite galaxies in the lowest stellar mass bin, i.e. $(1-2) \times 10^{10} \mathrm{M}_{\odot}$. The red (blue) lines represent the satellites around the most (least) massive primary galaxies. Therefore, these distributions are the equivalent of the leftmost blue and red data points in the topmost panels of Fig. 6. We emphasize that the uncertainties derived from the individual spectra are very large. The figure tests the hypothesis that the observed trends could be an artefact of the stacking procedure, where the signal would be originating only from a few spectra, either because of their higher $\mathrm{S} / \mathrm{N}$ or the presence of significantly different EWs. The distributions are shown following a standard Gaussian kernel density estimator (with a kernel size $\sigma / 5$, where $\sigma$ is the standard deviation of the distribution). The individual measurements reveal the same trend as in the stacked data, confirming the trends are robust against stacking artefacts.

In order to translate these EW measurements into physical parameters, we apply a simple comparison of the data with a grid of simple stellar populations (see Section 4). Note that since we are only using a reduced set of observations - the noise level and flux calibration of the GAMA/AAT spectra prevent us from applying full spectral fitting methods - we restrict the analysis to SSP-equivalent values of age (Fig. 8) and metallicity (Fig. 9). The top panels give the values along with the $1 \sigma$ uncertainty, with the same colourcoding as in Fig. 6. The orange lines in the bottom panels give the difference between the two extreme cases in red and blue, shown in the top panels. In this figure, we add to our (EW-based) estimates, those from the SED fitting analysis based on MAGPHYS (da Cunha, Charlot \& Elbaz 2008), where Fig. 8 includes the constraint on the mass- $(M)$ and SDSS- $r$-band luminosity-weighted $(L)$ age. The MAGPHYS and the EW-based constraints originate from independent information about the galaxies. The former uses the continuum, measured through broad-band photometry over a wide wavelength range (Driver et al. 2016), whereas this study targets a reduced set of age- and metallicity-sensitive line strengths. The results are compatible, with age differences involving pairs in lower and higher mass primary galaxies as large as $\sim 1-2 \mathrm{Gyr}$, although the spectral analysis is more sensitive to small age differences. This environmentrelated effect decreases with increasing secondary mass. The variations with respect to metallicity are negligible, although we note that the index plots (Fig. 6) give a significant separation of metallicitysensitive indices, such as $[\mathrm{MgFe}]^{\prime}$, with respect to primary mass. The likelihood analysis - marginalizing over all possible values of the population parameters - washes out this information, but at the low-mass end, the EW trends (Fig. 6) suggest a trend towards more metal-rich satellites when located close to more massive galaxies.

Fig. 10 compares the structural parameters of satellite galaxies as a function of stellar mass. We use the Sérsic decomposition of the surface brightness profiles presented in Kelvin et al. (2012). On the left, the top panel shows the projected effective radius in physical 
(A) $\mathrm{M}_{\mathrm{PRI}}$ selection

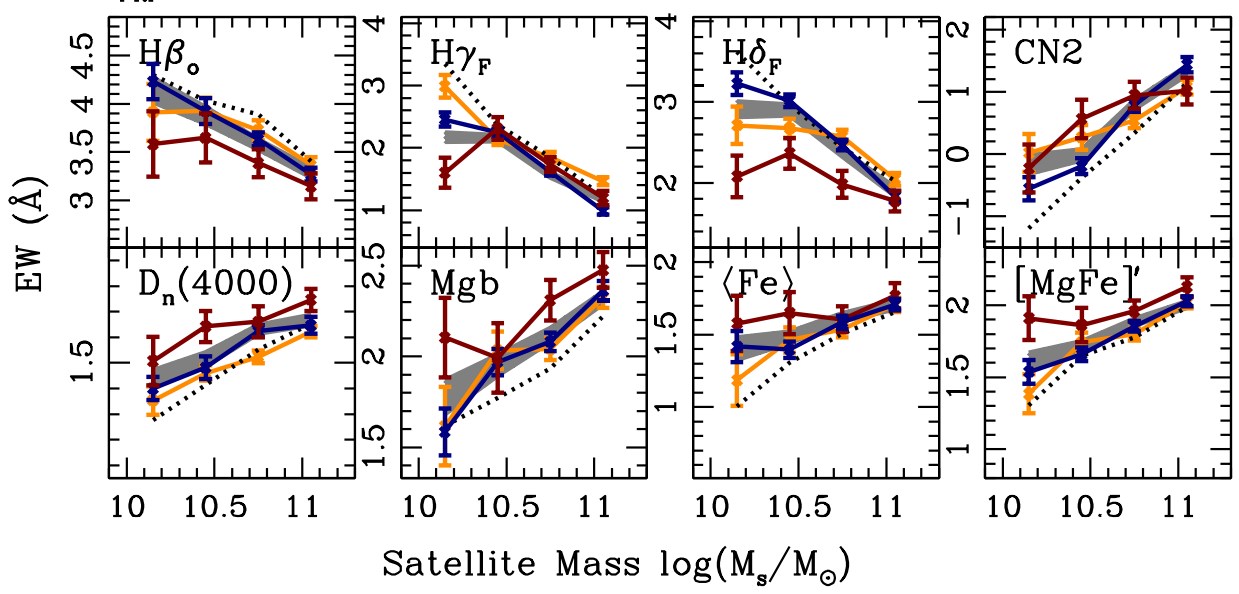

(B) $\mathrm{G}^{3} \mathrm{C}$ selection (luminosity-based)

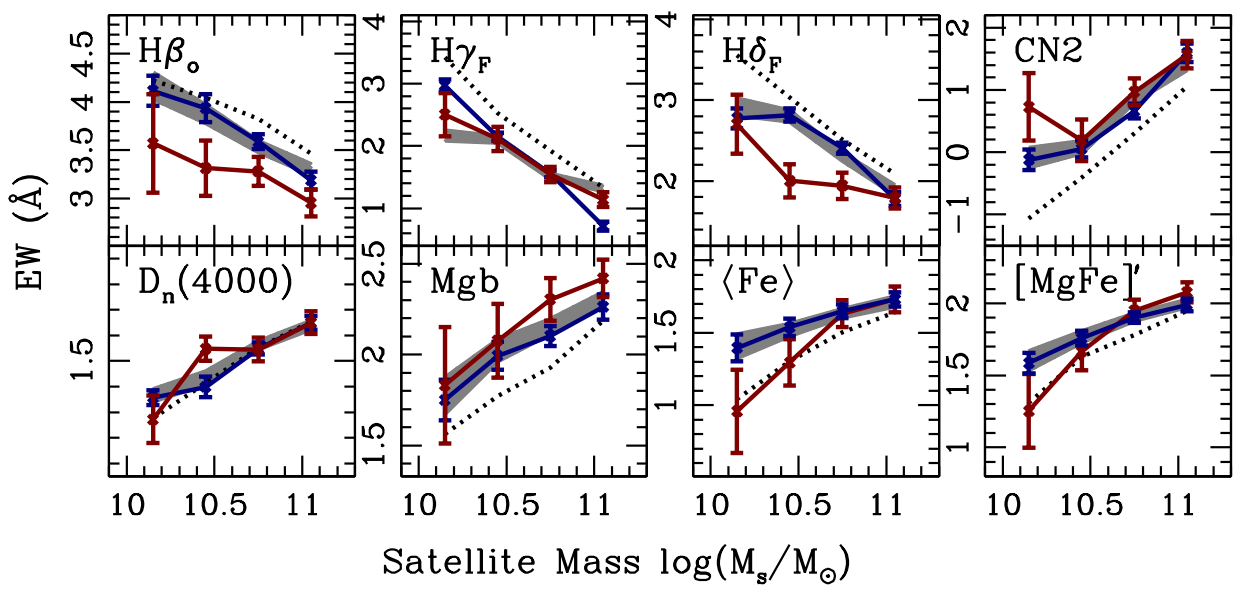

(C) $\mathrm{G}^{3} \mathrm{C}$ selection (dynamical)

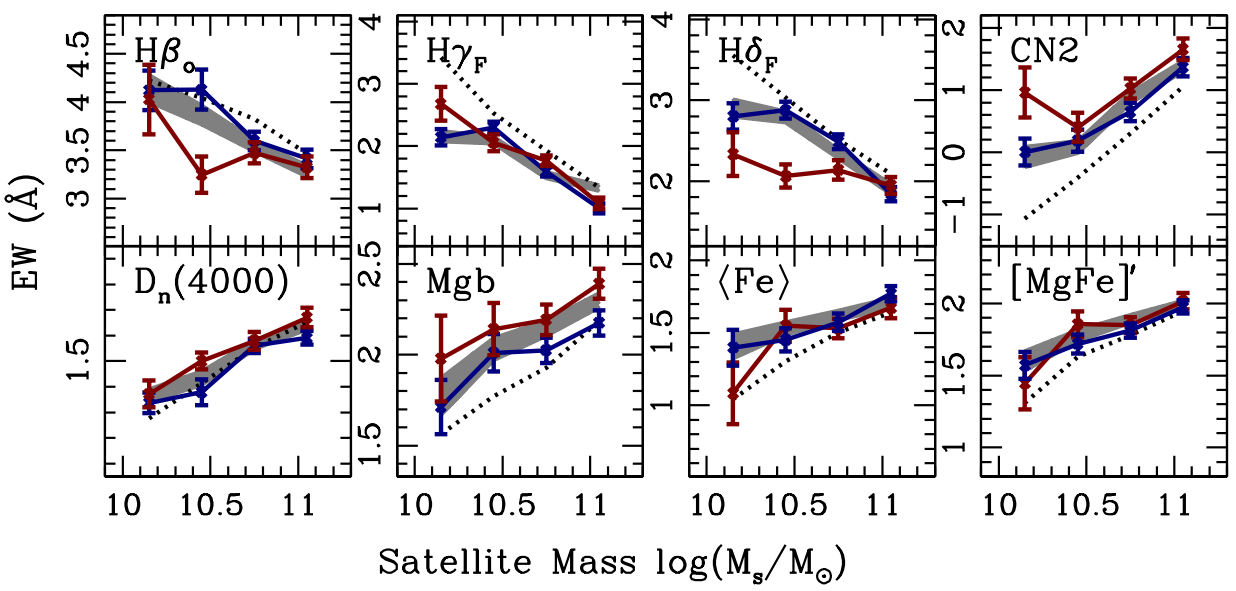

Figure 6. Equivalent widths of stacked spectra of satellite galaxies in close pairs, shown as a function of the stellar mass of the galaxy, and a second, environment-related parameter, namely the mass of the primary galaxy (A; top panels) or the group mass (B, C; middle and bottom panels) according to the $\mathrm{G}^{3} \mathrm{C}$ catalogue of Robotham et al. (2011). The grey shaded area is the trend for stacks made irrespective of the environment parameter. The blue (red) data points are the values for satellites around the least (most) massive primary galaxy, or group mass, corresponding to the bottom and top rows in the grids shown in Fig. 3. The orange points in the top panels represent a subsample comprised of systems where the closeness criterion is tighter (see the text for details). The dotted black line corresponds to the large sample from all GAMA spectra (i.e. stacked with the same criterion as with the satellite galaxies regarding S/N, but irrespective of whether they are located in close pairs). The halo masses in (B) are derived from the total group luminosity, whereas in (C), the masses are derived from a dynamical argument (see the text for details). 

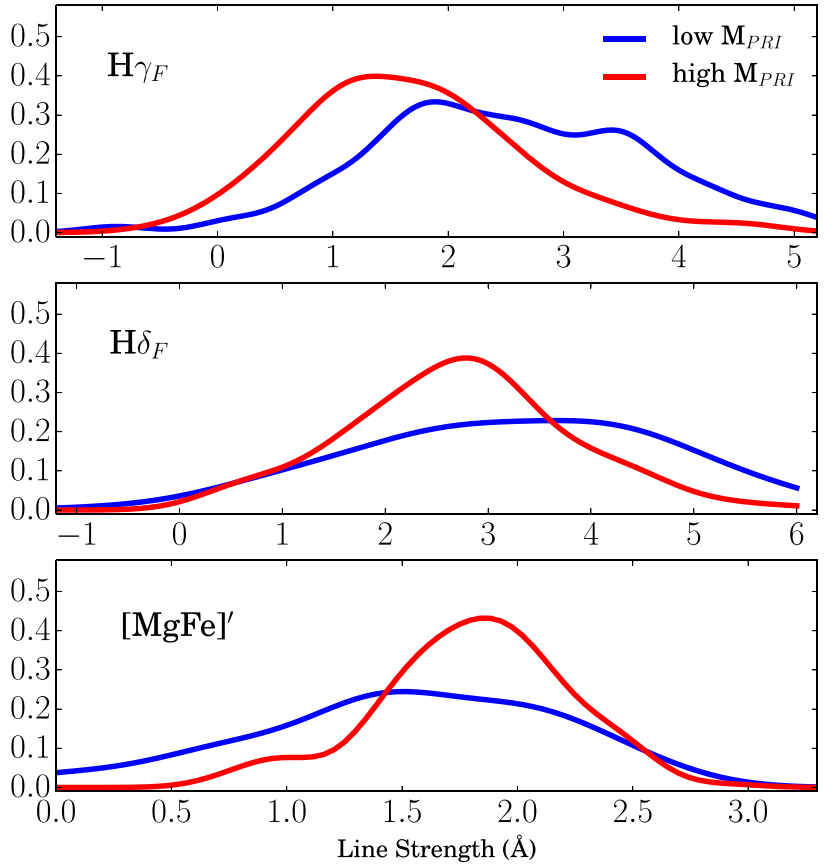

Figure 7. Distribution of equivalent widths measured in individual spectra. They correspond to satellite galaxies in the lowest stellar mass bin, stacked according to primary mass (shown in red/blue, as labelled). They show that the differences found in the stacked measurements are representative of the whole sample, and are not affected by a few spectra with higher $\mathrm{S} / \mathrm{N}$ or deeper line strengths.

units, and the bottom panel gives the Sérsic index. The sample of satellite galaxies used in the spectral stacking - i.e. avoiding those that featured low $\mathrm{S} / \mathrm{N}$, fringed spectra or with $n_{\mathrm{Q}}<3-$ is shown as grey dots. The blue and red symbols give the average and (rms) scatter of subsamples binned with respect to stellar mass, corresponding to satellites close to the most (red) or least (blue) massive primary. The aim of this figure is to assess whether the stacked data represent structurally different systems. The figure discards this hypothesis. Within the scatter, both subsamples correspond to similar types of galaxies, and no significant systematic would be expected from this issue. On the right, the histograms show the distribution of the Sérsic index (top panel) and the effective radius (bottom panel) for the sample of primary (i.e. massive) galaxies. Different histograms correspond to subsamples split with respect to halo mass or the presence of a satellite. No significant differences can be found, except for a weak trend towards lower Sérsic indices in primary galaxies without a satellite, or in groups with lower halo masses. The histograms show that the effects on the stellar populations cannot be caused by a morphology-related selection bias.

\subsection{Population differences in massive primary galaxies}

In addition to the analysis of the populations in satellites, we extend the study to their massive companions. As these are massive systems $-\log M_{\mathrm{s}}>10^{11} \mathrm{M}_{\odot}-$ we expect a rather homogeneous population of red-sequence galaxies. Fig. 11 shows the trends of the EWs in stacked spectra of primary galaxies, as a function of their stellar mass. For reference, we include the shaded region of Fig. 6, which represents the massive end of the satellite sample. Analogously to the satellite sample, three different stacking criteria are pursued: On the top panels (labelled A), the red (blue) lines correspond to stacks of primary spectra with (without) a nearby satellite. On the middle and bottom panels (labelled B and C), the red (blue) lines are the results for primary galaxies in the most (least) massive groups, according to the $\mathrm{G}^{3} \mathrm{C}$ catalogue, following the same criteria as in Fig. 3. The preparation of the stacked spectra follows the same methodology as for the satellite sample (Section 3.2), although the adopted fiducial velocity dispersion is $250 \mathrm{~km} \mathrm{~s}^{-1}$. In (A), the Balmer line indices show a trend towards older populations in galaxies with a satellite (red), consistent with the higher $4000 \AA$ break. The trend appears stronger in $D_{n}(4000)$. One could expect this result from the fact that Balmer indices are mostly sensitive to recent episodes of formation (within $\lesssim 1-2 \mathrm{Gyr}$ ), whereas $D_{n}(4000)$ varies over a wider range of stellar ages (see e.g. Kauffmann et al. 2003). However, the interpretation of the $4000 \AA$ break index gets more complicated in old populations such as those

(A) $\mathrm{M}_{\mathrm{PRI}}$ selection

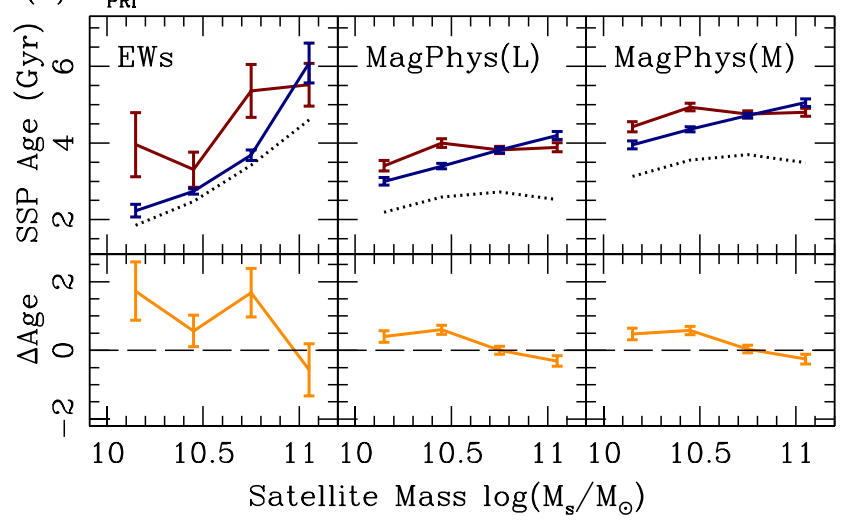

(B) $\mathrm{G}^{3} \mathrm{C}$ selection

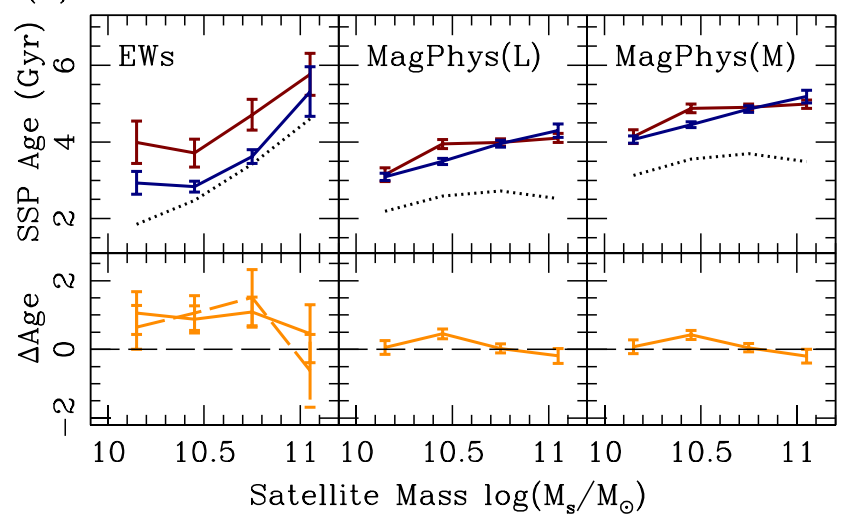

Figure 8. Age comparison from the line strength analysis (left-hand panel) and MagPhys (middle and right-hand panels). The blue/red colour-coding is the same as in Fig. 6. The dotted black line corresponds to the large sample comprising all high S/N GAMA spectra within the same redshift window. The orange lines in the bottom panels show the age difference between the red and the blue lines in the top panels, i.e. between satellites next to the most and the least massive primary galaxies. The leftmost panels (A) represent the stacked data according to the stellar mass of the primary, whereas the rightmost panels (B) correspond to stacks following the (luminosity-based) halo mass. We also include a dashed orange line representing the age difference when binning with respect to the dynamical halo mass estimates. 


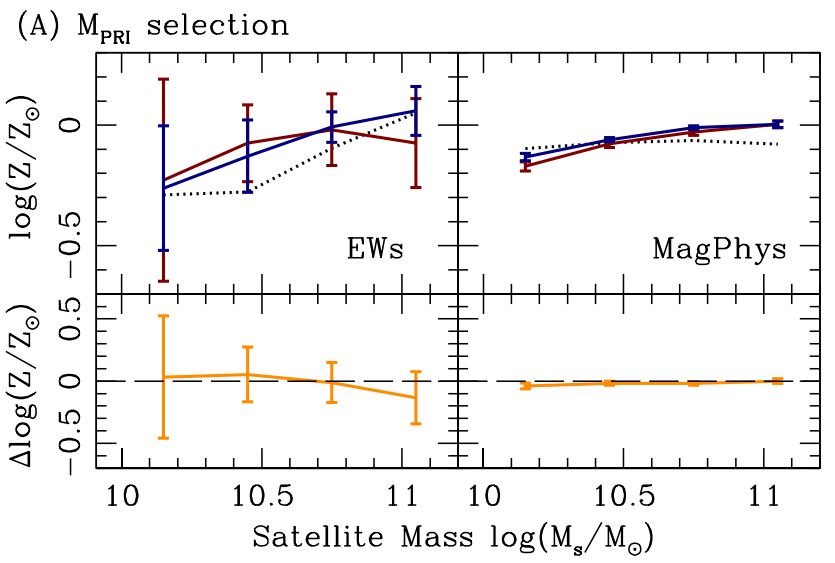

(B) $\mathrm{G}^{3} \mathrm{C}$ selection

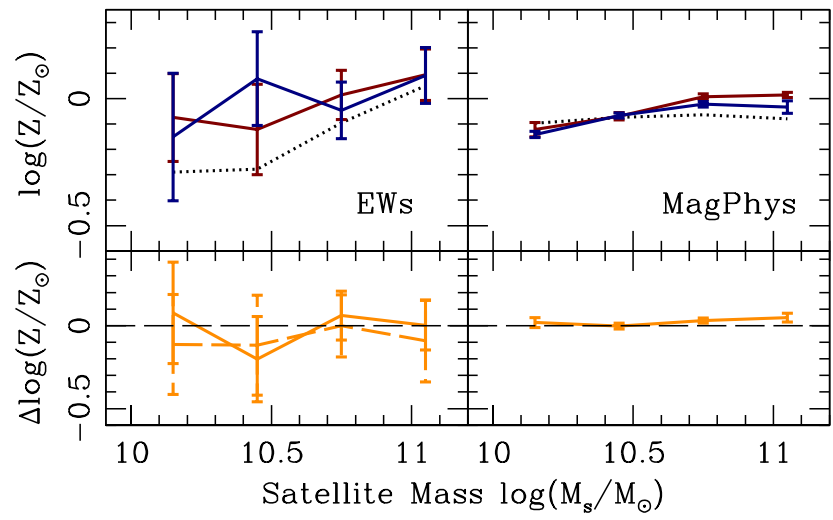

Figure 9. Metallicity comparison from the line strength analysis and with MagPhys. The notation is the same as in Fig. 8.
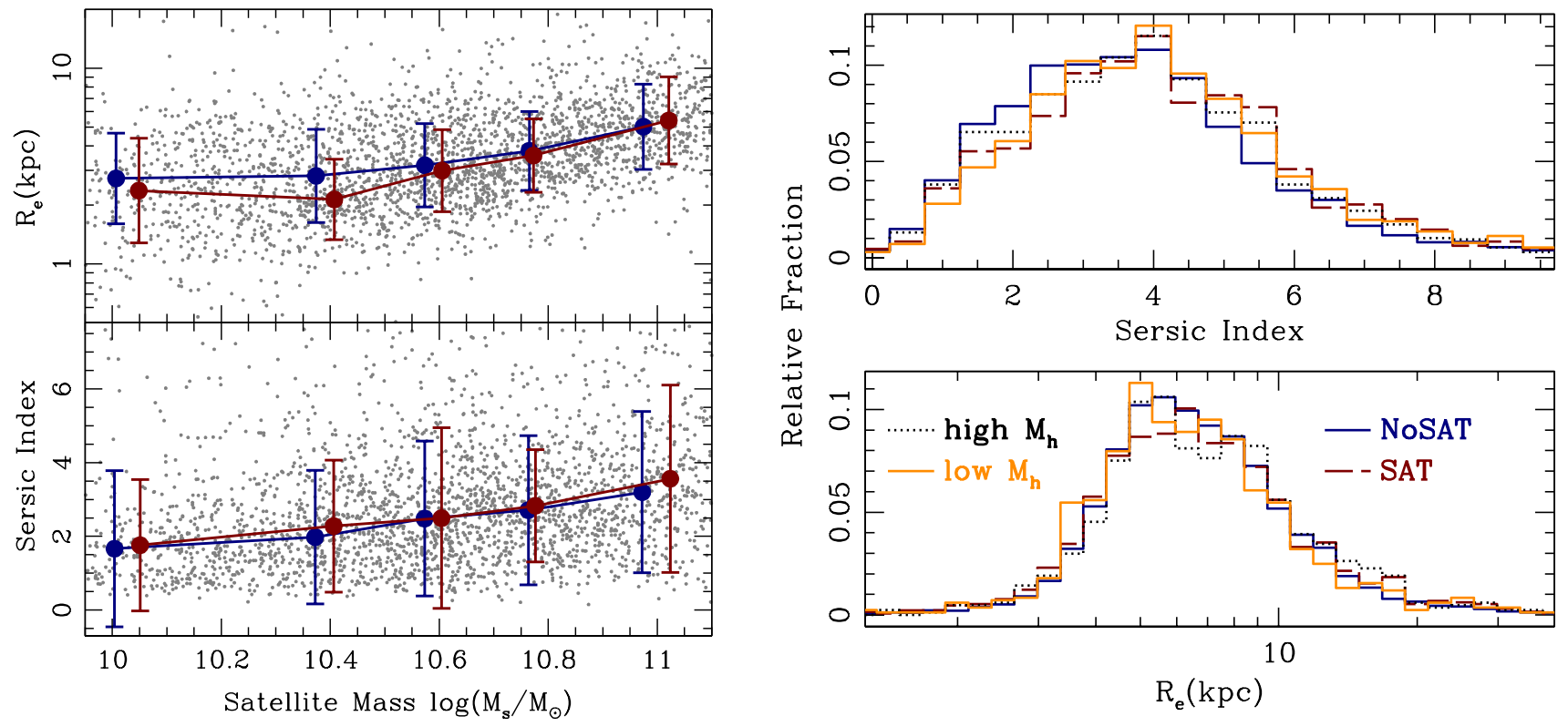

Figure 10. Left-hand panel: distribution of structural parameters in the sample of satellite galaxies. The effective radius (top panel) and Sérsic index (bottom panel) is presented as a function of the stellar mass for the sample of satellite galaxies. Grey dots correspond to individual galaxies, whereas the dots and error bars are averages and (rms) scatter for data binned at fixed number of galaxies per bin. The red (blue) symbols correspond to satellites close to the most (least) massive primary galaxies, i.e. the same criterion as in, for example. Fig. 6. Right-hand panel: distribution of structural parameters for the primary galaxies, split with respect to the presence of a satellite or in halo mass. For the latter, we only choose primaries where the luminosity-derived halo mass lies in the lowest quartile (low $M_{\mathrm{h}}$ ) or the highest quartile (high $M_{\mathrm{h}}$ ).

expected in massive galaxies, where the degeneracy with respect to metallicity is more pronounced. The metallicity indices $\mathrm{Mgb}$ and $[\mathrm{MgFe}]^{\prime}$ also show an increased strength in those primary galaxies with a nearby satellite, whereas $\langle\mathrm{Fe}\rangle$ does not show any variation. In (B) and (C), segregated with respect to group mass, some differences are apparent, although weaker, except for $\mathrm{CN} 2$, which has a pronounced variation with the luminosity-weighted halo masses. This result is consistent with the environment-related trends found in this index in Carretero et al. (2007). The metallicity differences (through $\mathrm{Mgb}$ and $[\mathrm{MgFe}]^{\prime}$ ) are also weak, with differences between the methodology used to derive halo masses. Thus, we conclude from this result that the populations of the massive primary galaxies appear homogeneous, with a small difference between primaries with and without satellites. A comparison of the $\mathrm{Mgb}$ and the $\langle\mathrm{Fe}\rangle$ line strengths in the topmost panels of Fig. 11 may suggest an overall $[\mathrm{Mg} / \mathrm{Fe}]$ enhancement in massive galaxies with a satellite. Following the simple proxy of $[\mathrm{Mg} / \mathrm{Fe}]$ presented in La Barbera et al. (2013), namely comparing the total metallicity when constraining the populations using either $\mathrm{Mgb}$ or $\langle\mathrm{Fe}\rangle$ as a metallicity indicator (instead of $[\mathrm{MgFe}]^{\prime}$ ), gives an overall enhanced population of the primary galaxies around $[\mathrm{Mg} / \mathrm{Fe}] \sim+0.2$ to $+0.3( \pm 0.1)$ dex, but no discernible difference - within error bars - between the stacks of primary galaxies with and without satellites. We expect the observed variations in $\mathrm{Mgb}$ and $\langle\mathrm{Fe}\rangle$, shown in Fig. 11, to be caused by a complex combination of age, metallicity and $[\mathrm{Mg} / \mathrm{Fe}]$ differences. The weaker trends in the group-selected stacks may be representative, but this issue is beyond the scope of this paper, leaving it to future work. 
(A) SAT/no SAT selection

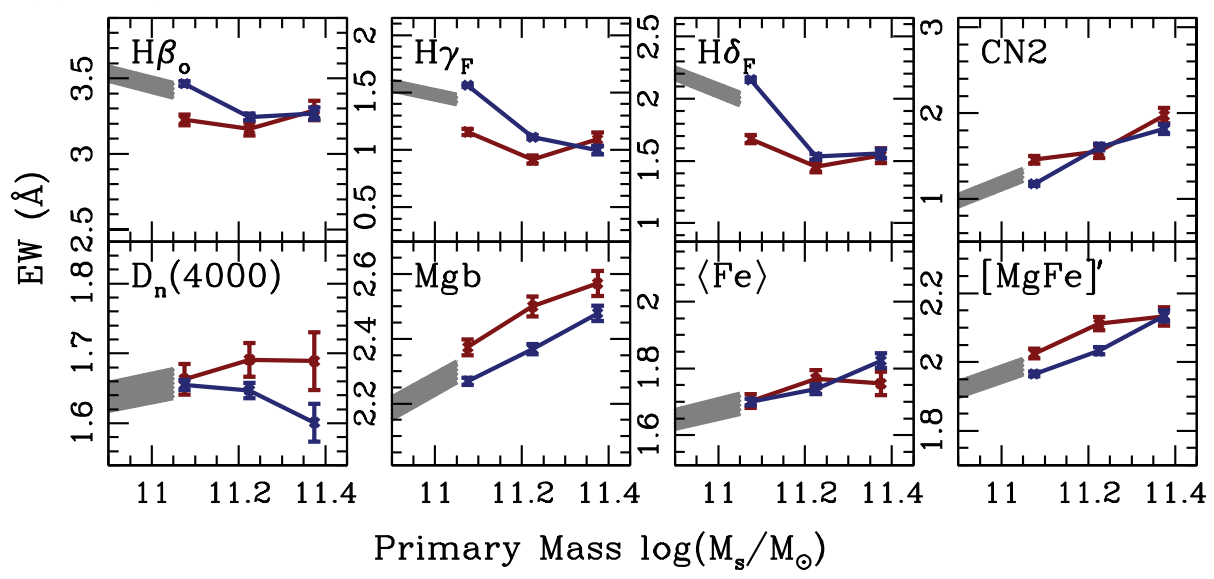

(B) $\mathrm{G}^{3} \mathrm{C}$ selection (luminosity-based)

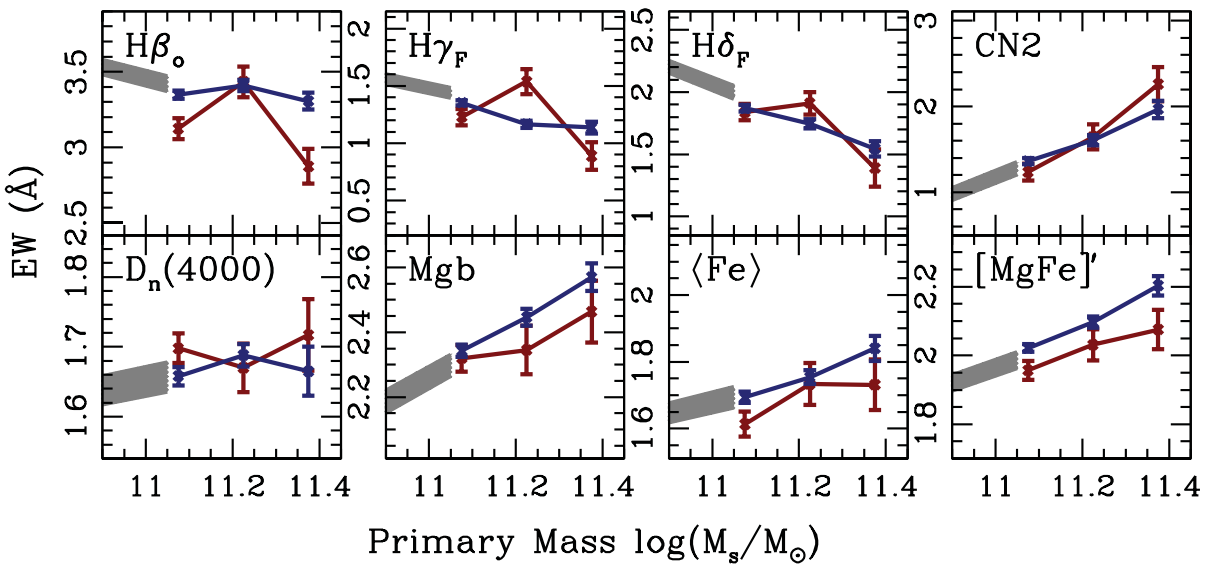

(C) $\mathrm{G}^{3} \mathrm{C}$ selection (dynamical)

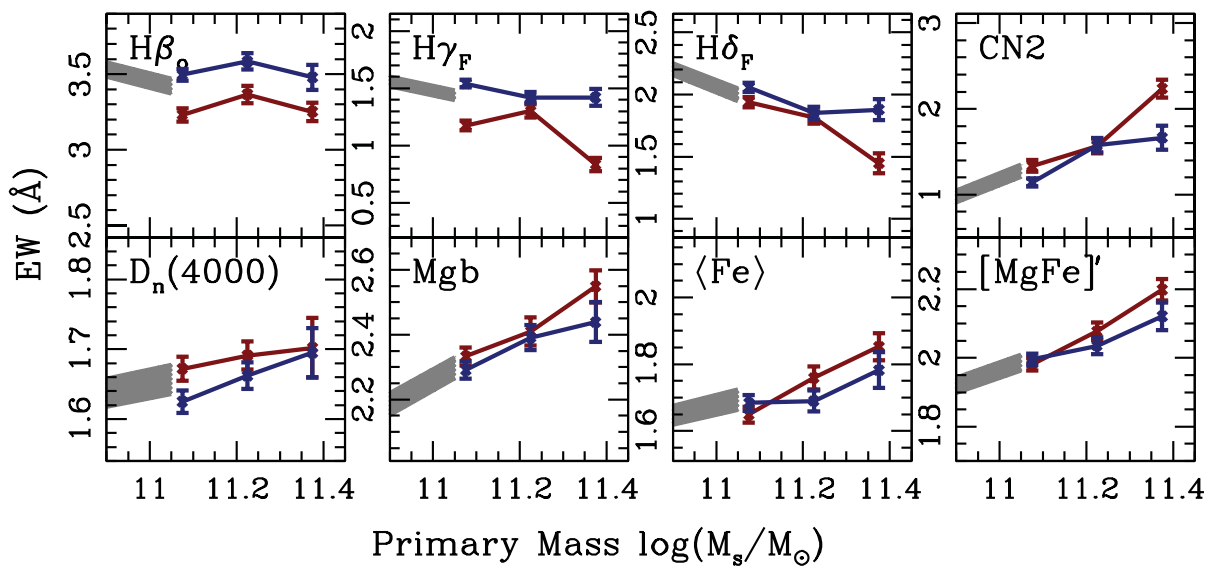

Figure 11. EWs of stacked spectra of primary galaxies, shown as a function of stellar mass. In (A) (top panel), the stacking criterion is based on whether the primary has a satellite (red) or not (blue). In (B) and (C) (middle and bottom panels), the spectra are stacked according to group mass, derived from the $\mathrm{G}^{3} \mathrm{C}$ catalogue (Robotham et al. 2011), using either the scaling relation between halo mass and total luminosity (B), or the dynamical mass (C). The red (blue) lines represent primary galaxies located in the highest (lowest) groups. The grey shaded region corresponds to the general trend in satellite galaxies, as shown in Fig. 6. See the text for details.

\subsection{Comparison with the close pair selection of Davies et al.}

Our results provide an independent approach to the evolution of close galaxy pairs with respect to Davies et al. (2015), who focused on star formation diagnostics, rather than on the under- lying stellar populations. They found star formation to be suppressed in secondary galaxies involving minor mergers. This is equivalent to the older populations found in satellites around the most massive primary galaxies (red lines at the low-mass end in Figs 6 and 8). However, we emphasize that stellar populations 
provide a cumulative (integral) picture of the past star formation history, whereas a star formation diagnostic gives the 'instantaneous' (differential) version of the same process, more specifically within the last $\sim 100$ Myr. Therefore, the study of Davies et al. (2015) is more sensitive to recent events triggering star formation, whereas this work reveals a deeper connection with environment over longer time-scales ( $\gtrsim 1 \mathrm{Gyr}$ ). These results are also consistent with the radial gradients found in massive earlytype galaxies, with a dominant old and metal-poor component in the outer regions (see e.g. La Barbera et al. 2011, 2012; Greene et al. 2015).

\section{SUMMARY}

Taking advantage of the uniform spatial completeness of the GAMA survey, we select a sample of dynamically close pairs involving at least one massive (stellar mass $\gtrsim 10^{11} \mathrm{M}_{\odot}$ ) galaxy, over the redshift range $0.1 \leq z \leq 0.3$. The study focuses on the stellar populations of the satellite galaxies through a targeted set of spectral features. Since the populations in the primary and secondary galaxies will eventually merge, this study provides insight on the dominant growth channel of massive galaxies during the so-called second, ex situ phase. Due to the S/N and flux calibration characteristics of the GAMA/AAT spectra, the analysis is based on a continuum-subtracted version of the data, stacking individual spectra according to two parameters: a 'local' observable - the stellar mass of the satellite, and an environment-related observable, using either the mass of the primary galaxy, or the group (i.e. dark matter halo) mass. In addition to the well-known local trend between age/metallicity and galaxy mass, we find a significant environmental trend in the stellar populations, so that at fixed mass, satellite galaxies linked to more massive primary galaxies appear older. This trend is especially apparent at the low-mass end of our sample (satellite mass $\sim 10^{10} \mathrm{M}_{\odot}$ ), where the SSP-equivalent age differences are 1-2 Gyr. This age difference decreases towards the more massive satellites, and it is most significant when considering either the mass of the primary or the group mass as the environment-related parameter. The data cannot disentangle the effect between these two. We emphasize that in contrast to the recent study of close pairs using star formation diagnostics (Davies et al. 2015), this work focuses on the stellar populations, providing insight into the star formation processes over longer time-scales. The consistency of these results reinforce the idea that galaxy-related processes due to the primary must play an important role on the observed differences, and that the trends with respect to group halo mass may be inherited from the intrinsic correlation between the two. Therefore, our results are consistent with the general picture of galactic conformity (Weinmann et al. 2006). The stellar mass of a galaxy is robustly found as the main indicator of the properties of its underlying stellar populations. However, at fixed stellar mass, the population of satellite galaxies have more in common with the corresponding central in its group. In this work, this conformity appears in the age of the stellar populations: If the primary galaxy is older (roughly more massive), the age of the secondary is older than that of another secondary with the same stellar mass, orbiting a less massive, thus younger, primary. Such a result is also consistent with the observed lack of age gradients in giant early-type galaxies (La Barbera et al. 2011, 2012). These observational trends should provide useful constraints for numerical simulations of galaxy formation, where the internal age and metallicity gradients are very sensitive to the subgrid physics (see e.g. Hirschmann et al. 2015).

\section{ACKNOWLEDGEMENTS}

IF gratefully acknowledges support from the AAO through their distinguished visitor programme in 2014 and 2016, as well as support from ICRAR and the Royal Society. MLPG acknowledges CONICYT-Chile grant FONDECYT 3160492. MSO acknowledges the funding support from the Australian Research Council through a Future Fellowship (FT140100255). Funding for SDSS-III has been provided by the Alfred P. Sloan Foundation, the Participating Institutions, the National Science Foundation, and the U.S. Department of Energy Office of Science. The SDSS-III web site is http://www.sdss3.org/. GAMA is a joint European-Australasian project based around a spectroscopic campaign using the AAT. The GAMA input catalogue is based on data taken from the SDSS and the UKIRT Infrared Deep Sky Survey. Complementary imaging of the GAMA regions is being obtained by a number of independent survey programmes including GALEX MIS, VST KIDS, VISTA VIKING, WISE, Herschel-ATLAS, GMRT and ASKAP providing UV to radio coverage. GAMA is funded by the STFC (UK), the ARC (Australia), the AAO and the participating institutions. The GAMA website is http://www.gama-survey.org/.

\section{REFERENCES}

Baldry I. K. et al., 2014, MNRAS, 441, 2440

Balogh M. L., Morris S. L., Yee H. K. C., Carlberg R. G., Ellingson E., 1999, ApJ, 527, 54

Brough S. et al., 2013, MNRAS, 435, 2903

Cardelli J. A., Clayton G. C., Mathis J. S., 1989, ApJ, 345, 245

Carretero C., Vazdekis A., Beckman J. E., 2007, MNRAS, 375, 1025

Cervantes J. L., Vazdekis A., 2009, MNRAS, 392, 691

Cid Fernandes R., Mateus A., Sodré L., Stasińska G., Gomes J. M., 2005, MNRAS, 358, 363

Conselice C. J., 2014, ARA\&A, 52, 291

da Cunha E., Charlot S., Elbaz D., 2008, MNRAS, 388, 1595

Daddi E. et al., 2005, ApJ, 626, 680

Davies L. J. M. et al., 2015, MNRAS, 452, 616

Davies L. J. M. et al., 2016, MNRAS, 455, 4013

Driver S. P. et al., 2011, MNRAS, 413, 971

Driver S. P. et al., 2016, MNRAS, 455, 3911

Falcón-Barroso J., Sánchez-Blázquez P., Vazdekis A., Ricciardelli E., Cardiel N., Cenarro A. J., Gorgas J., Peletier R. F., 2011, A\&A, 532, A95

Ferreras I. et al., 2014, MNRAS, 444, 906

Greene J. E., Janish R., Ma C.-P., McConnell N. J., Blakeslee J. P., Thomas J., Murphy J. D., 2015, ApJ, 807, 11

Han J. et al., 2015, MNRAS, 446, 1356

Hartley W., Conselice C. J., Mortlock A., Foucaud S., Simpson C., 2015, MNRAS, 451, 1613

Hawkins K., Jofré P., Gilmore G., Masseron T., 2014, MNRAS, 445, 2575

Hirschmann M., Naab T., Ostriker J. P., Forbes D. A., Duc P.-A., Davé R., Oser L., Karabal E., 2015, MNRAS, 449, 528

Hopkins A. M. et al., 2013, MNRAS, 430, 2047

Jiang C. Y., Jing Y. P., Han J., 2014, ApJ, 790, 7

Kauffmann G. et al., 2003, MNRAS, 341, 33

Kawinwanichakij L. et al., 2016, ApJ, 817, 9

Kelvin L. S. et al., 2012, MNRAS, 421, 1007

Kitzbichler M. G., White S. D. M., 2008, MNRAS, 391, 1489

Kroupa P., 2001, MNRAS, 322, 231

La Barbera F., Ferreras I., de Carvalho R. R., Lopes P. A. A., Pasquali A., de la Rosa I. G., De Lucia G., 2011, ApJ, 740, L41

La Barbera F., Ferreras I., de Carvalho R. R., Bruzual G., Charlot S., Pasquali A., Merlin E., 2012, MNRAS, 426, 2300

La Barbera F., Ferreras I., Vazdekis A., de la Rosa I. G., de Carvalho R. R., Trevisan M., Falcón-Barroso J., Ricciardelli E., 2013, MNRAS, 433, 3017 
Lackner C. N., Cen R., Ostriker J. P., Joung M. R., 2012, MNRAS, 425, 641

Le Fèvre O. et al., 2000, MNRAS, 311, 565

Liske J. et al., 2015, MNRAS, 452, 2087

López-Sanjuan C. et al., 2012, A\&A, 548, A7

Mármol-Queraltó E., Trujillo I., Pérez-González P. G., Varela J., Barro G., 2012, MNRAS, 422, 2187

Mármol-Queraltó E., Trujillo I., Villar V., Barro G., Pérez-González P. G., 2013, MNRAS, 429, 792

Naab T., Johansson P. H., Ostriker J. P., 2009, ApJ, 699, L178

Newman A. B., Ellis R. S., Bundy K., Treu T., 2012, ApJ, 746, 162

Oser L., Naab T., Ostriker J. P., Johansson P. H., 2012, ApJ, 744, 63

Patton D. R., Carlberg R. G., Marzke R. O., Pritchet C. J., da Costa L. N., Pellegrini P. S., 2000, ApJ, 536, 153

Pérez-González P. G. et al., 2013, ApJ, 762, 46

Robothamn A. S. G. et al., 2010, Publ. Astron. Soc. Aust., 27, 76

Robothamn A. S. G. et al., 2011, MNRAS, 416, 2640

Robothamn A. S. G. et al., 2014, MNRAS, 444, 3986

Rogers B., Ferreras I., Kaviraj S., Pasquali A., Sarzi M., 2009, MNRAS, 399,2172

Rogers B., Ferreras I., Peletier R., Silk J., 2010, MNRAS, 402, 447

Ruiz P., Trujillo I., Mármol-Queraltó E., 2014, MNRAS, 442, 347
Sánchez-Blázquez P. et al., 2006, MNRAS, 371, 703

Schlafly E. F., Finkbeiner D. P., 2011, ApJ, 737, 103

Taylor E. N. et al., 2011, MNRAS, 418, 1587

Thomas D., Maraston C., Bender R., 2003, MNRAS, 339, 897

Trager S. C., Worthey G., Faber S. M., Burstein D., González J. J., 1998, ApJS, 116, 1

Trujillo I. et al., 2006, ApJ, 650, 18

Trujillo I., Conselice C. J., Bundy K., Cooper M. C., Eisenhardt P., Ellis R. S., 2007, MNRAS, 382, 109

Trujillo I., Ferreras I., de La Rosa I. G., 2011, MNRAS, 415, 3903

van Dokkum P. G. et al., 2010, ApJ, 709, 1018

Vazdekis A., Ricciardelli E., Cenarro A. J., Rivero-González J. G., DíazGarcía L. A., Falcón-Barroso J., 2012, MNRAS, 424, 157

Viola M. et al., 2015, MNRAS, 452, 3529

Weinmann S. M., van den Bosch F. C., Yang X., Mo H. J., 2006, MNRAS, 366,2

Worthey G., Ottaviani D. L., 1997, ApJS, 111, 377

This paper has been typeset from a $\mathrm{T}_{\mathrm{E}} \mathrm{X} / \mathrm{LT} \mathrm{E} \mathrm{X}$ file prepared by the author. 